\title{
POTENCIALIDAD PARA EL DESARROLLO DE MODALIDADES DEL TURISMO AVENTURA (BOULDERING Y COASTEERING) EN TANDIL, ARGENTINA
}

\author{
Aldo G. Ramos \\ UNICEN. Tandil, Argentina ${ }^{1}$ \\ Facultad de Ciencias Humanas \\ Centro de Investigaciones y Estudios Ambientales
}

(Fecha envío: 28/08/17 - Fecha aceptación: 09/12/17)

\begin{abstract}
Resumen
En los últimos años se observa un crecimiento del denominado turismo alternativo y dentro de este del turismo aventura, asociado a un aumento de las actividades deportivas por parte de un segmento importante de la población, que incluye prácticas extremas con un elevado nivel de riesgo. Entre estas aparecen el bouldering y el coasteering como segmentos crecientes en algunos países europeos. El objetivo del trabajo es analizar si determinadas características existentes en la localidad de Tandil (Argentina) podrían permitir ampliar la oferta existente incluyendo estas modalidades. Considerando las condiciones físicas, el desarrollo de la oferta actual y acciones de capacitación en turismo aventura es posible potencialmente incluir a las modalidades mencionadas en el desarrollo turístico del destino.
\end{abstract}

Palabras claves

turismo aventura, bouldering, coasteering, áreas mineras, turismo alternativo

\section{Abstract}

In recent years there is a growth of the so-called alternative tourism and within this adventure tourism, associated with an increase in sporting activities by a large segment of the population, which includes extreme practices with a high level of risk. Among these are bouldering and coasteering as growing segments in some European countries. The objective of the work is to analyze whether certain characteristics in the town of Tandil (Argentina) could allow to expand the existing offer including these modalities. Considering the physical conditions, the development of the current offer and training actions in adventure tourism is possible to potentially include the modalities mentioned in the tourist development of the destination.

\section{Key words}

adventure tourism, bouldering, coasteering, mining areas, alternative tourism

\section{INTRODUCCIÓN}

El denominado turismo alternativo que abarca un amplio espectro de modalidades y cuyos principales exponentes son el ecoturismo, el turismo rural y el turismo aventura, comenzó a desarrollarse con fuerza en las últimas tres décadas aproximadamente (a pesar que el turismo rural en Francia se iniciara a mediados de siglo para reducir el éxodo a las ciudades y por necesidad de retorno de la población urbana a espacios más naturales) con la generación de productos turísticos vinculados a experiencias que incorporaban con fuerza a la naturaleza y a las comunidades locales.

Estas modalidades que fueron surgiendo se inscriben en los procesos de transformación del turismo tanto desde su dimensión productiva como sociológica. En el primer caso, el modelo alternativo de turismo se vincula a un modelo donde la flexibilidad, la segmentación del mercado, la búsqueda de innovación en los productos, de transformación de la estacionalidad y la estandarización y la revisión de los procesos de armado, gestión y venta cobran cada vez más fuerza. Estos cambios afectan a los distintos sistemas de la vida social, entre ellos el consumo y por supuesto tendrán su influencia en el turismo. Una sociedad industrial avanzada, se transforma hacia un nuevo modelo denominado de formas diversas (sociedad red, sociedad digital, sociedad de tercera ola), pero que cuyo término más extendido es el de postmodernidad.

\footnotetext{
${ }^{1}$ Profesor Adjunto de Turismo Sustentable y Turismo Alternativo. Licenciado en Geografía. Master en Ecoauditorias y Planificación Empresarial del Medio Ambiente.
} 
En el marco de esta sociedad postmoderna surgen, por tanto, unos valores sociales que van a configurar una demanda turística plural en las sociedades receptoras y que lleva a la reorganización de los productos en el ocio y el turismo y de todas sus conductas sociales. "En este nuevo marco aspectos como el desarrollo de las tecnologías, la globalización, la nueva cultura de lo sustentable y el compromiso en el consumo, el hedonismo, la búsqueda del placer y de las nuevas experiencias, el individualismo, la diferenciación y la búsqueda de la calidad, están claramente ligadas a la configuración de nuevas demandas en cuanto al entorno, el destino, la vivencia, esto es, en cuanto al producto turístico deseado". (Fernández Alvariño, C. 2010:12).

Las nuevas formas de turismo asociadas al turismo alternativo combinan bienes, recursos y experiencias en un territorio dado, desarrollándose hasta nuestros días. En dicho contexto aparece, paulatinamente, un listado amplio de tipologías conceptuales (definidos o no) asociadas a esta modalidad y a las actividades allí comprendidas.

Estos cambios sociales producidos incluyen la necesidad de desarrollar actividades físicas, para el cuidado del cuerpo, pero rápidamente se fueron incorporando prácticas que implican un esfuerzo mayor, surgiendo así los denominados deportes extremos, por ejemplo. Una sociedad que comienza a sentir la necesidad de autosuperarse, de exigirse aún más, pero en un esquema de logros personales básicamente y no de manera competitiva y profesional.

Así comienza a desarrollarse el turismo aventura, el cual cuenta con una amplia gama de actividades, desde las más sencillas (hikking) hasta las complejas que requieren entrenamiento físico y mental y equipamiento e instructores especialmente entrenados (montañismo).

\section{CARACTERÍSTICAS, DEFINICIONES Y NUEVAS MODALIDADES DEL TURISMO AVENTURA}

Desde un punto de vista conceptual podemos decir que, si bien el escenario natural es de primordial importancia para la oferta del turismo aventura, también la actividad que se realice debe ofrecer una oportunidad para que los participantes experimenten emociones diferentes, esfuerzos físicos y sensaciones de riesgo.

Como expresa Ewert (en Sung, H. 1996) lo que distingue a estas actividades del turismo de aventura de las recreaciones tradicionales al aire libre es la búsqueda deliberada del riesgo y la incertidumbre del resultado generalmente denominado aventura" donde un individuo por lo general enfrenta cada vez más "riesgos" y "amenazas personales.

El concepto de riesgo o riesgo percibido, asociado a la superación personal es esencial para las actividades de turismo de aventura, por lo cual podemos decir que sin este componente existiría una reducción fuerte de la satisfacción del turista. A esto también se asocia la idea de competencia percibida y desafío personal, con metas más abstractas y autoimpuestas.

Como expresan De Azevedo y de Farias (2013:67) el riesgo percibido contempla dos aspectos: incertidumbre sobre los resultados y sobre las consecuencias. De acuerdo con Campbell y Goodstein (citado en De Azevedo y de Farias, 2013:67) el riesgo percibido es mayor a medida que el grado de incertidumbre aumenta y/o existe la posibilidad de que se produzcan consecuencias negativas.

De esta forma el riesgo percibido implica que la adquisición de determinado servicio, en este caso de turismo aventura, puede tener ciertas consecuencias negativas, por ejemplo, no poder realizar cumbre por parte de un escalador a causa de condiciones meteorológicas extremas. Estas condiciones, aumentan el riesgo en la práctica de la actividad y podrían llevar a situaciones que terminen en accidentes o incluso la muerte. Por esta razón es un segmento sumamente particular y se debe diferenciar de actividades desarrolladas en espacios totalmente controlados con mínima preparación.

De todas formas, no estamos hablando de suicidas o masoquistas por lo cual, si bien esto requiere de argumentaciones propias de la psicología, el riesgo es un concepto relativo asociado a las habilidades del turista para desarrollar la actividad, traducido en por ejemplo número de ascensos a montañas, a la experiencia de los guías y al equipamiento utilizado. Estas dos últimas variables son analizadas por los participantes a través de una exhaustiva búsqueda de información.

Considerando lo expresado, las principales características del turismo aventura son:

- Se requiere de instrucción previa para la práctica de la actividad, especialmente en la operación de equipos con eficiencia y seguridad. Implica acompañamiento de personal calificado en la operación (guía especializado).

- Debe cumplir parámetros claros de seguridad según cada actividad, que deben ser garantizados por quien contrata los servicios con el turista, por quien opera la actividad y especialmente el guía. El destino turístico debe contar con dotación, servicios e infraestructura acorde con la actividad, especialmente para atención de emergencias. 
- Se desarrolla más fácilmente con viajeros independientes o en circuitos organizados para grupos de tamaño reducido.

- Incluye un factor de riesgo por la característica particular o desafío que impone la actividad; este riesgo es controlado por la operación y competencia del personal que la desempeña.

- Tiene un ánimo competitivo, cuando se asocia a una práctica deportiva.

- Es especializado como producto turístico, exigiendo capacidades técnicas para su prestación y una alta segmentación del mercado.

- El turismo de aventura debe compartir los principios del desarrollo sustentable, cumpliendo especialmente requisitos ambientales y de vinculación de comunidades locales.

De acuerdo con Araújo Vila et al (2011) las actividades dentro del turismo aventura se pueden clasificar en:

- Actividades terrestres: Área de marcha, Alpinismo/escalada, Marcha a caballo, Ciclomontañismo, Espeleología, Cicloturismo, Puenting, Barranquismo, Esquí de travesía, Esquí de fondo, Raquetas, Perros con trineo, Motos de nieve, Tiro con arco, 4x4, Outdoor training,

- Actividades acuáticas: Piragüismo/kayak, Turismo fluvial, Buceo/submarinismo, Surf/Windsurf/Kitesurf, Motonáutica, Rafting, Hidrobob/Hidrospeed, Vela, Esquí acuático.

- $\quad$ Actividades aéreas: Ala delta, Globo aerostático, Parapente, Paracaidismo de pendiente, Paracaidismo, Ultraligeros, Vuelo sin motor, Heliexcursión,

A partir de estas características y de los tipos de actividades podemos considerar algunas de las definiciones elaboradas en relación al turismo aventura como la de Hall (1989) que la conceptualiza como "un amplio espectro de actividades turísticas al aire libre, que se comercializan por lo general y que suponen una interacción con el ambiente natural lejos del hogar del participante y que contiene elementos de riesgos donde el participante, el escenario y el manejo de la experiencia del participante influencian en el desenlace" o la de Sung (1996:5) para quien es "la suma de los fenómenos y de las relaciones que surgen de las interacciones de las actividades turísticas de aventura con el medio ambiente natural lejos del área de residencia habitual del participante y que contiene elementos de riesgos en el que el resultado o desenlace está influenciado por la participación, escenario y organizador de la experiencia del turista".

En definitiva, puede señalarse entonces que el turismo aventura está atravesado por procesos de transformación generales asociados a la postmodernidad y al sistema económico del post-fordismo que vertebran al turismo alternativo, como un modelo que incluye las experiencias de aventura. Es de suponer entonces que, analizando los fundamentos del turismo alternativo, sus dimensiones, se deduzca que las actividades de aventura deben presentar con mayor o menor fuerza estos principios, elementos o componentes. Destacando el rol de las actividades como atractivos, fuertemente asociados al modelo de comportamiento alocéntrico o mediocéntrico del turista. Los primeros se caracterizan por ser extrovertidos, complejos y que están realizando actividades constantemente y que lo que más valoran es la libertad y la independencia. Conciben el turismo como una acumulación de experiencias basadas en la aventura y en el riesgo, para lo cual planifican mínimamente el viaje para que de manera flexible se adapte a las condiciones de este. Eligen especialmente espacios principalmente agresivos como montañas, selvas, desiertos y que estén deshabitados o habitados por poblaciones que tienen escaso contacto con el resto de la sociedad. En el otro grupo encontramos turistas con intereses especiales porque conciben el turismo como el desarrollo de sus aficiones y entretenimientos, muy relacionados con actividades deportivas o de esfuerzo físico, asumiendo y deseando nuevas experiencias y aventuras, siempre y cuando el riesgo este controlado.

Los cambios en la sociedad han generado que el turismo aventura haya crecido fuertemente en los últimos años, producto del aumento de la práctica de actividades deportivas por parte de la población, de la necesidad de autosuperación como característica de la sociedad posmoderna y a un mayor contacto con la naturaleza y con otras comunidades.

De esta forma, según el informe Adventure Tourism Development Index de 2016 de la ATTA (Adventure Travel Trade Association), este segmento de turismo genero 263.000 millones de dólares, teniendo un crecimiento anual del 65\% entre 2009 y 2012, con un aumento de la inversión por parte del turista de casi el $20 \%$ en ese mismo periodo.

El reporte incluye análisis de tres regiones (Europa, Norteamérica y Sudamérica), que representan en conjunto casi el $70 \%$ de los viajes internacionales, de acuerdo con datos de la OMT (Organización Mundial del Turismo). Los datos del estudio mencionado reafirman la tendencia al crecimiento del turismo internacional y el papel de los viajes de aventura en esa evolución.

Según la OMT pueden existir dos tipos de actividades de aventura: por un lado tenemos las denominadas "soft" que son las que se realizan de manera más informal y con menos dedicación de tiempo y esfuerzo, con el deseo de disfrutar de la actividad o del atractivo natural con cierta comodidad y por otro lado podemos encontrar las actividades "hard" que implican un interés especial o una fuerte dedicación y la voluntad de disfrutar del entorno o de la práctica en condiciones de menor comodidad e incluso con un ánimo competitivo, sin llegar a la competencia deportiva. 
En el primer caso debería discutirse si realmente estamos frente a lo que podemos considerar como turismo aventura con las características que este implica o si son actividades deportivas y recreativas al aire libre con un cierto vínculo con el medio.

Según el informe Adventure Tourism Market Report (ATTA 2013), la mayor parte de este crecimiento se dará en el primer tipo de actividades de turismo aventura (soft adventure) con prácticas como hiking, kayaking, rafting, snorkeling, turismo de voluntariado y expediciones arqueológicas y en una menor cantidad, también se ampliarán las actividades de hard adventure (actividades de aventura más duras), como la escalada, el montañismo, la espeleología, heli-skiing, kitesurfing, trekking, paragliding, el bouldering y el coasteering.

\subsection{Bouldering}

La escalada en bloque o bouldering, consiste en escalar bloques de roca o pequeñas paredes, que pueden ser de hasta 6 u 8 metros de altura, sin la necesidad de los materiales de protección convencionales de la escalada (por ejemplo, cuerda, arnés, elementos de fijación, etc.) se realiza de lado y subiendo muy poco. La roca presenta "problemas" a resolver, tanto en ascenso como en travesía (horizontal). Sus inicios se remontan a principios del siglo XX, pero fue a mediados del siglo pasado cuando se popularizo dentro del montañismo.

En este tipo de escalada prima la dificultad extrema durante trayectos muy cortos. Como mecanismo de seguridad se utiliza una colchoneta portátil denominada crash pad y la pericia y atención de quienes deben cuidar al escalador para que la posible caída no tenga consecuencias de gravedad.

Este tipo de actividad requiere de un excelente estado físico y de mucha fuerza en los brazos y en las manos. Una de las problemáticas que presenta esta modalidad es la necesidad de limpiar las rocas por donde se va desarrollando la actividad para evitar que se desprendan restos o incluso la necesidad de eliminar plantas que puedan dificultar el paso del escalador, por esto es necesario eliminar selectivamente para no deteriorar el medio y con ello preservar la zona para futuras generaciones de escaladores o incluso otros usos.

Para el desarrollo de la actividad los bloques son señalizados y la organización debe comprobar la resistencia de los agarres. También es necesario limpiar las zonas de posibles caídas a fin de no dejar ningún objeto peligroso como, piedras o equipos de los participantes. En estas zonas se colocan los denominados crash pads, que tienen por objetivo amortiguar la caída del escalador en caso de producirse.

Normalmente los bloques de rocas utilizados tienen un lugar por el cual se puede bajar caminando.
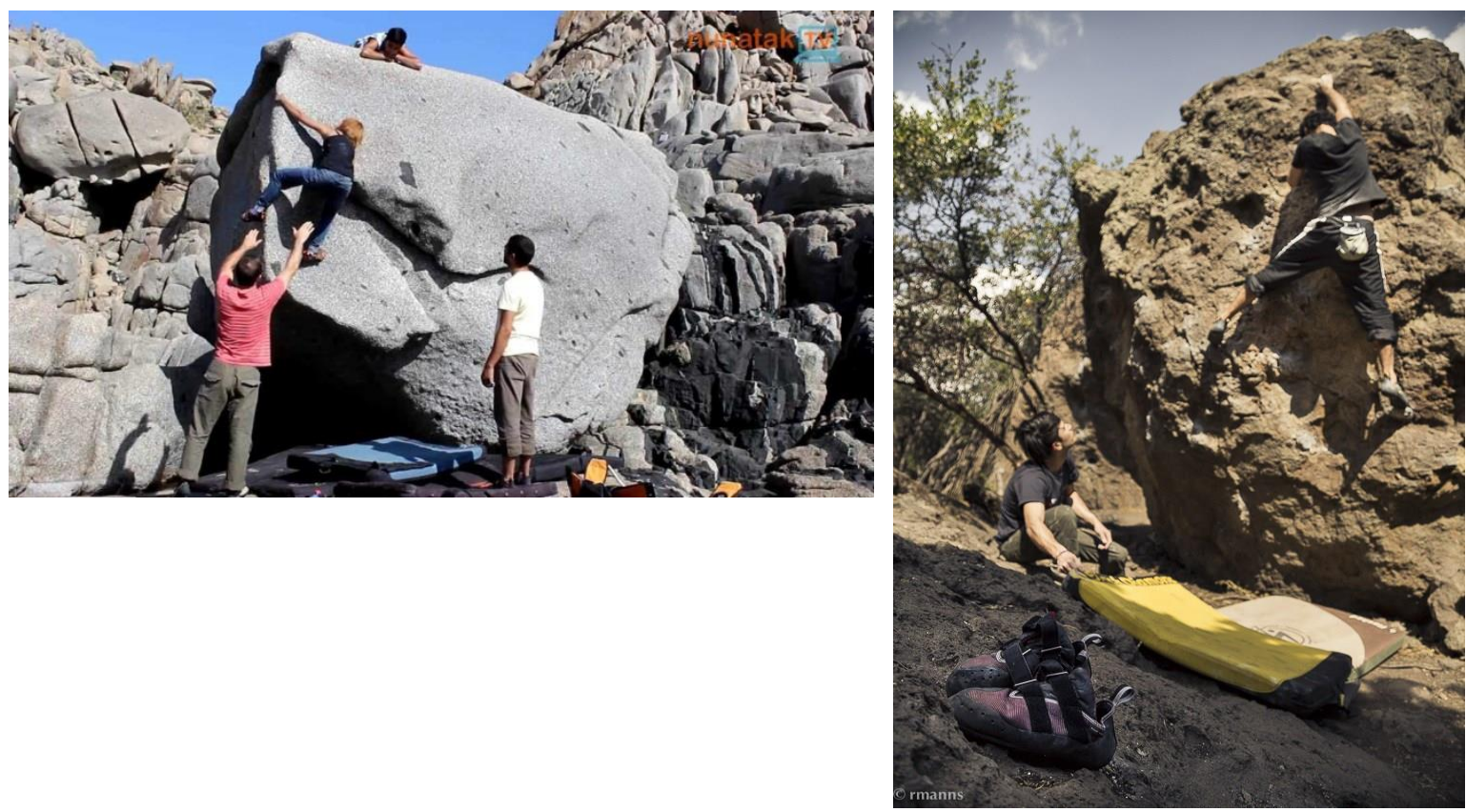

Imagen 1: Desarrollo del bouldering con escaladores y zona de seguridad

Fuente: http://www.dav.cl. extraído del sitio en marzo de 2017 


\subsection{Coasteering}

El coasteering, como explican Silva y Almeida (2011) es un deporte de aventura que consiste en realizar un recorrido costero combinando senderismo, saltos de altura al agua (hasta 8 metros), exploración de cuevas terrestres, buceo, escalada de travesía, rápel y puente de cuerda entre otras actividades. Según la South West Watersports (2004) es una actividad recreativa que atrae a personas variadas cuya motivación principal para algunas es la visita de lugares prácticamente inaccesibles y de belleza natural escénica y otras la búsqueda de emociones fuertes y adrenalina.

Una de las primeras utilizaciones de este término apareció en el libro Sea Cliff Climbing de 1973 pero es a partir de 1990 cuando esta modalidad comenzó a crecer en el Reino Unido teniendo en la costa galesa de Pembrokeshire uno de los espacios favoritos por sus características geomorfológicas.

El nivel de dificultad varía dependiendo de factores como el estado del mar, el terreno, la temperatura del agua, de la atmosfera, el viento y la extensión del recorrido. Aunque todos estos factores son importantes el que aparece como determinante es el estado del mar que implica dirección de corrientes, turbulencia en las rocas, etc. De acuerdo a estos elementos existen tres niveles posibles de dificultad, de menor a mayor: la Blue line, la White line y la Red line.

La actividad implica conocimientos previos de los participantes, organizándose grupos no mayores a 20 personas, aunque se considera que 12 es el número ideal considerando la relación con los guías y para reducir el potencial impacto ambiental, asociado al pisoteo de las rocas y efectos sobre la fauna y flora local, lo cual debe evitarse rotando las rutas y restringiendo las zonas con comunidades vulnerables. También es imprescindible que los guías estén entrenados especialmente y el uso de equipamiento especial (trajes de neoprene, calzado adherente, equipo de rescate, etc.)

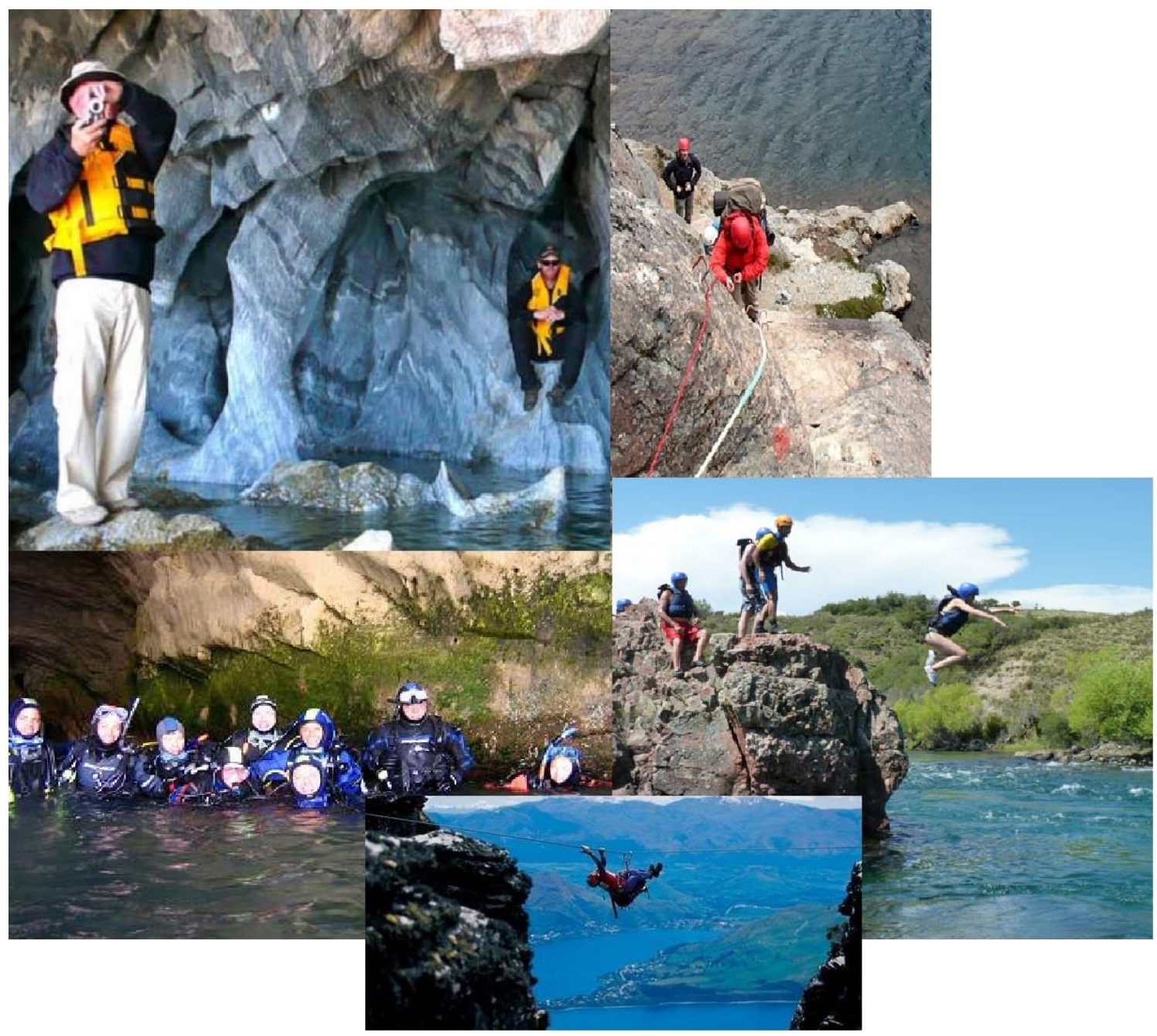

Imagen 2: Desarrollo de las distintas actividades que componen el coasteering

Fuente: Elaboración personal en base a promociones de la actividad en destinos europeos 


\section{METODOLOGÍA Y OBJETIVO}

El objetivo del trabajo es evaluar las condiciones (físicas y recursos humanos) de un destino de Argentina (Tandil) para poder desarrollar dos modalidades del turismo aventura que tienen algunos años en países europeos como son el bouldering y el coasteering y que podrían ampliar la oferta existente sobre turismo aventura complementándose con las existentes.

Es una investigación de tipo exploratoria, ya que permitirá examinar la temática de estas nuevas modalidades del turismo aventura que presentan un nulo desarrollo en el área de estudio y escaso a nivel del país permitiendo así acercarse a fenómenos turísticos nuevos o poco conocidos lo cual posibilita establecer prioridades para futuras investigaciones y estudios a posteriori, buscando identificar variables a desarrollar. (Hernández, R., Fernández, C., y Baptista, P. (2010). Metodología de le investigación, México D.F.: McGraw-Hill Interamericana).

La metodología se desarrolló a partir de:

a) La revisión de la bibliografía existente sobre las actividades de turismo aventura (bouldering y coasteering)

b) La evaluación general de las características naturales del destino a partir de trabajos previos realizados (Diagnóstico de sitios de interés geominero para el desarrollo del turismo: el caso del sistema serrano de Tandilia. Argentina, en International Journal of Scientific Management and Tourism (2017) 3-4: pp. 513-533; Geodiversidad, patrimonio minero y geoturismo: propuesta de parque geominero en Argentina, en Turismo y Sociedad. Vol. 17 julio-diciembre de 2015. pp. 17-37 y Pasivos ambientales mineros en la trama urbana: estudios de la ciudad de Tandil. Argentina, en Nadir. Rev. electrónica de geografía austral. Universidad Autónoma de Chile. ISSN: 0718-7130. Año 5, N. 2 agosto - diciembre 2013).

b.1) para el caso del coastering son: presencia de cuerpos de agua con costas recortadas y con altura en la costa, pero sin necesidad de grandes extensiones para nadar; características propicias del agua como temperatura del agua, visibilidad, calidad y el acceso a los lugares.

b.2) para el caso del bouldering son: presencia de bloques de roca con alturas aproximadas a los 8 metros ubicados en espacios accesibles y con mínimos obstáculos en las bases.

c) Análisis de la existencia de recursos humanos necesarios para desarrollar las actividades.

\section{ANÁLISIS DE CASO: TANDIL, PROVINCIA DE BUENOS AIRES, ARGENTINA}

El área de estudio, Tandil, se localiza en el sureste de la Provincia de Buenos Aires (República Argentina) y se encuentra a $350 \mathrm{Km}$. de la ciudad de Buenos Aires la cual concentro según datos de la Encuesta de Ocupación Hotelera de Argentina el 23,3\% del movimiento turístico de 2017, calculado en 10.135 .729 turistas. Tandil tiene una población actual que supera los 120.000 habitantes. El Municipio está emplazado en la Pampa Húmeda y más precisamente, en el sistema serrano de Tandilia, que lo atraviesa en sentido NO-SE.

Este sistema orográfico se halla caracterizado por un conjunto de serranías bajas y de formas redondeadas en el centro y norte del partido, mientras que cambian a formas de mesas o tabulares hacia el oeste y sur. Con respecto al emplazamiento, la ciudad se extiende de forma irregular, en una superficie aproximada de $50 \mathrm{~km}^{2}, \mathrm{y}$ cuyo soporte se caracteriza por ser una encrucijada de valles que descienden desde las Sierras de Tandil hacia la llanura Norte, continuándose hasta la depresión del Río Salado.

El cordón serrano representa una provincia fisiográfica, constituida por sierras, cerros, cerrilladas y lomas que sobresalen entre 50 y 250 metros de la llanura pampeana. Alineado en sentido NO-SE por algo más de $300 \mathrm{~km}$, su ancho es variable, siendo menor (unos seis $\mathrm{km}$ ) en ambos extremos y mayor en la parte central (unos $60 \mathrm{~km}$ en la zona de la ciudad de Tandil). En el área de estudio, el basamento granítico de las sierras y cerros presentan formas redondeadas, que han sido comparadas con cúpulas, domos y conos, asociados al Basamento Cristalino Precámbrico. 


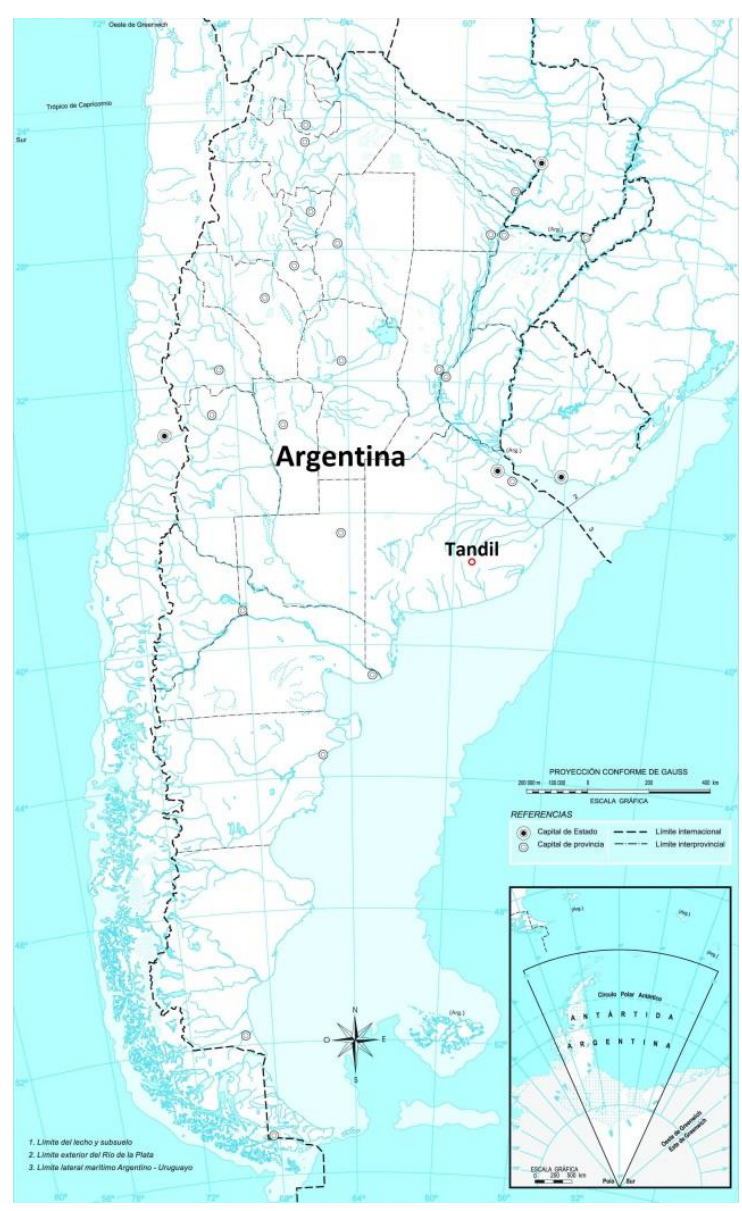

Mapa 1: Ubicación del área de estudio

Fuente: Elaboración personal. Mapa base extraído de www.vmapas.com

\subsection{Factores que posibilitarían el desarrollo del bouldering y coasteering en Tandil}

En primer lugar, el paisaje de la zona en estudio es un elemento singular, asociado a formaciones vegetales, a partir de la dominancia de diferentes tipos o formas biológicas, reconociendo en el área diferentes fisonomías tales como pastizales, cultivos y malezales; arbustales y formaciones arbóreas (montes de abrigo, de ribera y forestaciones).

Estas características naturales singulares dentro de la región han permitido el crecimiento de actividades al aire libre, convirtiendo a la localidad en un destino donde es posible realizar turismo aventura. Pero también en los alrededores de la ciudad de Tandil, existen numerosas explotaciones mineras a cielo abierto. Algunas de ellas son muy antiguas y se iniciaron hace más de 100 años con el auge de los picapedreros (inmigrantes europeos), que formaron parte de la historia, cultura y arquitectura de la ciudad de Tandil.

Un segundo aspecto a considerar son las características geomorfológicas y geológicas del sistema serrano que, por su uso minero, permitió el desarrollo de numerosas canteras que se encuentran actualmente en su mayoría abandonadas y ostentan frentes abiertos de diferentes altura y estabilidad e incluso cuerpos de agua permanentes en las cavas, en los cuales se pueden realizar actividades de aventura específicas (entre ellas escalada, rapel, tirolesa o actividades de buceo en menor proporción).

Si bien como se concluyó en una investigación anterior (Ramos y Fernández, 2015) se estableció que las actividades de turismo aventura en Tandil podrían enmarcarse principalmente dentro de la categoría señalada por la OMT como suaves, realizadas de manera más informal y con una menor dedicación, disfrutando de la actividad o del atractivo natural desde un punto de vista paisajístico, pero con cierta comodidad. También se observó que bajo la modalidad de aventura se ofertan actividades al aire libre y en contacto con la naturaleza y con experiencias lúdico-deportivas tradicionales, para grupos grandes y segmentos estandarizados que son propias de un turismo convencional y no de un modelo alternativo.

Pero pese a que existan ciertas contradicciones entre la teoría y la práctica en el desarrollo de la actividad, en parte producto de la falta de definiciones del sector púbico y de las características del turista promedio, es posible decir que la oferta de turismo aventura existe y es posible encauzar a la misma siguiendo los parámetros 
de calidad de servicio y ambiental que esta modalidad a nivel internacional exige. Sumando a la oferta existente encontramos otras modalidades que si bien están orientadas a un público especifico, están en crecimiento como por ejemplo el desarrollo de actividades de buceo en una cava de una cantera inundada.

La forma de las sierras obedece a varios procesos que actúan simultáneamente, siendo los principales la meteorización química que predomina sobre la meteorización física y la erosión. La alteración química de la roca madre comienza por los bloques diaclasados. La descomposición progresa hacia el centro formando capas concéntricas de roca más blanda. Esta forma de disgregación se denomina exfoliación esferoidal y produce cuerpos redondeados en los que, delgadas capas pueden ser separadas del núcleo esférico central, como puede observarse en varios lugares de la zona serrana. Algunos de estos bloques pueden utilizarse para el bouldering que como fue explicado anteriormente implica el uso de bloques grandes de roca con una altura que ronda como máximo los 6 u 8 metros de altura para escalar.

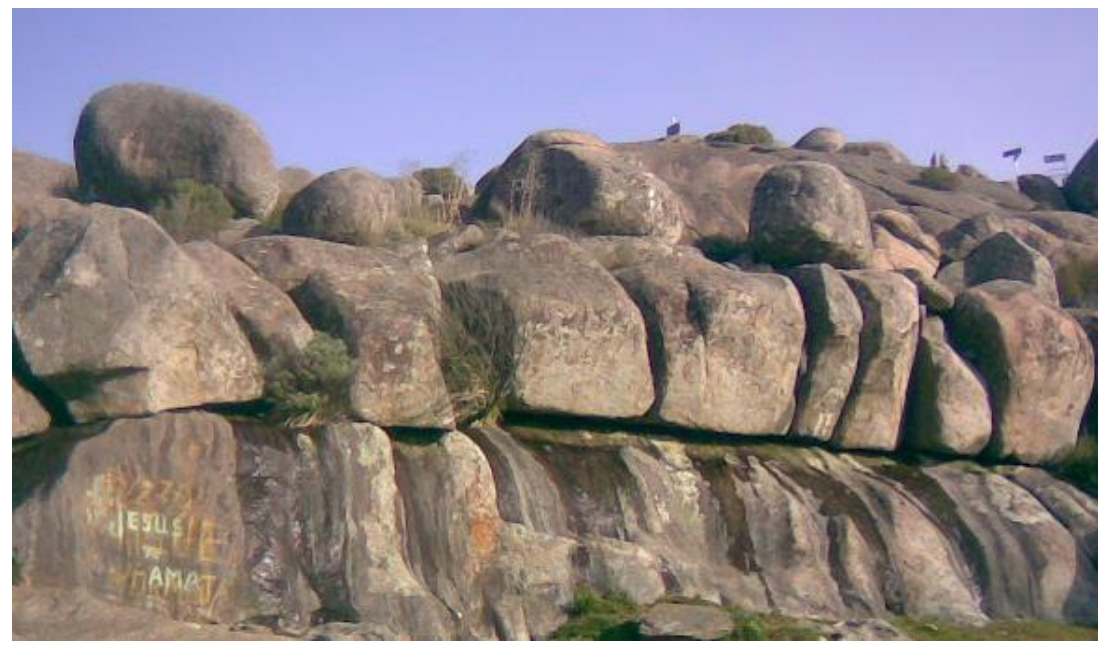

Imagen 3: Formación de bloques redondeados por la meteorización Fuente: Archivo personal. Año 2017

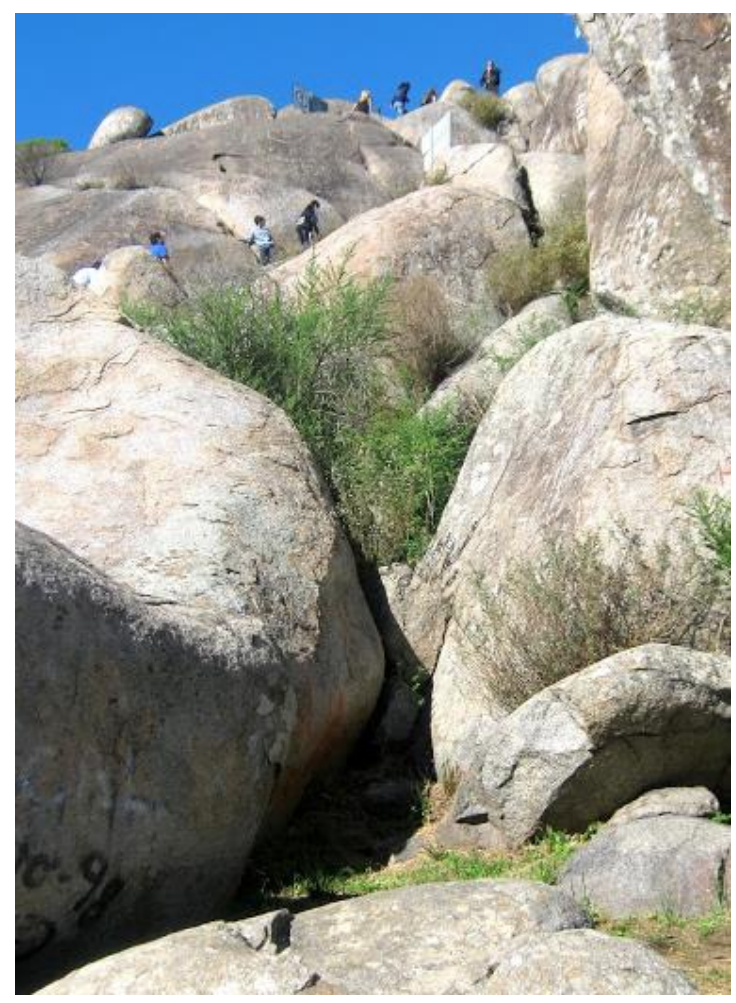

Imagen 4: Actividad de hikking en sector serrano Fuente: Archivo personal. Año 2017 
Pero además del uso del espacio serrano natural y considerando las características del bouldering, básicamente respecto a los posibles impactos que las tareas de limpieza pueden ocasionar, es posible reutilizar muchos de los bloques desprendidos por la actividad minera o incluso reubicando otros, permitiendo así evitar impactos en áreas poco transformadas del sector serrano y reutilizar espacios ya transformados por la minería. Incluso como son espacios que presentaron alguna transformación producto del trabajo de maquinaria para acceder al lugar y para trasladar el material existente, es más sencillo tener espacios limpios donde colocar pads para reducir los efectos de las potenciales caídas, logrando así una mejor organización de la actividad. El uso de estos espacios para actividades de rapel y escalada se viene desarrollando desde hace años, por lo cual es factible poder sumar prácticas de escalada libre, incluso no serían actividades que competirían ya que las alturas de las zonas a utilizar son distintas.
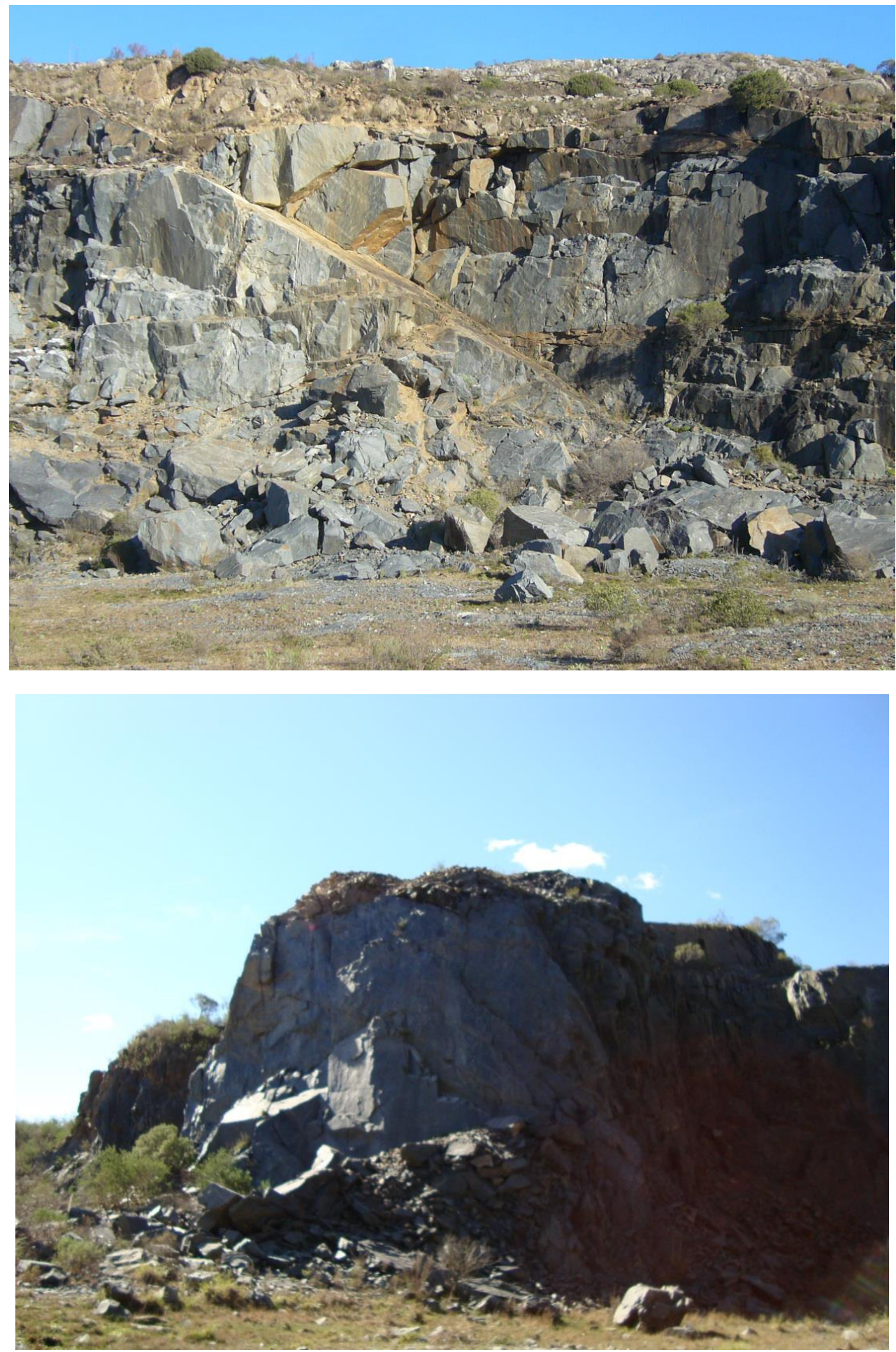

Imagen 5 y 6: Área minera donde pueden observarse bloques y cortes factibles de uso para bouldering Fuente: Archivo personal. Año 2016 
Para el caso del coasteering si bien no existen lagos o ríos con caudal importante, las cavas de las canteras que se inundaron ofrecen la posibilidad de realizar las actividades propias de esta modalidad, con la excepción de que no existe el riesgo por los movimientos de las corrientes, que en el caso de la costa de Gales por ejemplo le otorga un nivel de dificultad importante. Pero exceptuando esto, las costas de estos cuerpos de agua creados por la actividad minera podrían permitir realizar los saltos al agua, ya que existen incluso paredes con más de 8 metros de altura, realizar rapel y escalada, senderismo por sus márgenes y observación de fauna y flora (nativa y exótica) que fue colonizando estas áreas transformadas por el hombre. El uso de estos espacios inundados requiere de estudios de calidad del agua, ya que, si bien tienen aporte continuo de lluvias e incluso del nivel freático, tienen muy poco movimiento por lo cual se debe monitorear el nacimiento de algas por perdida de oxigenación o contaminación por sustancias vertidas sin control de áreas urbanizadas aledañas. Aunque cabe aclarar que es reducida la permanencia de los participantes en el agua, ya que en este sentido lo que se busca fundamentalmente es la generación de adrenalina a partir del salto, no involucrando buceo ni natación de largos recorridos.

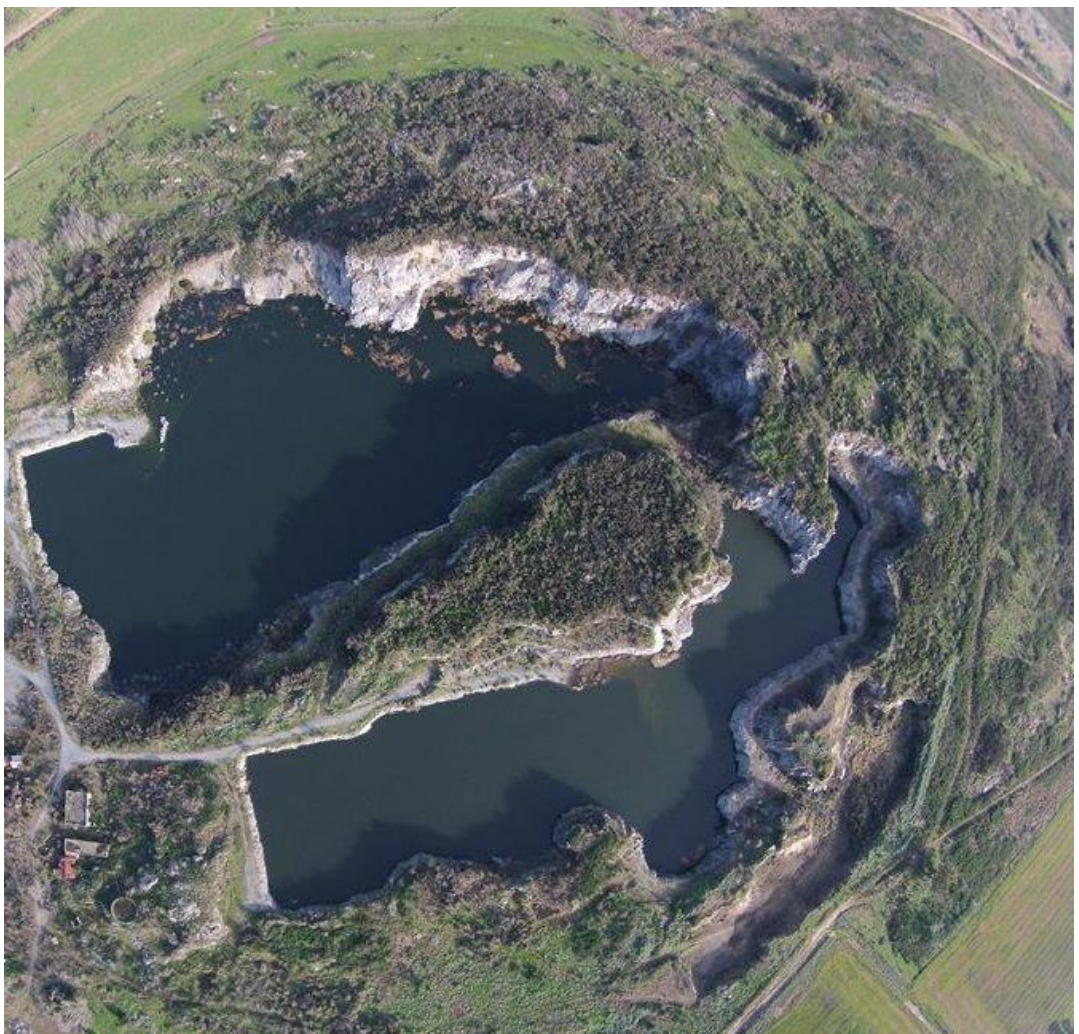

Imagen 7: Vista aérea de una de las cavas inundadas de forma permanente donde puede observarse la costa recortada que podría ser factible de uso para el coasteering.

Fuente: Google Earth

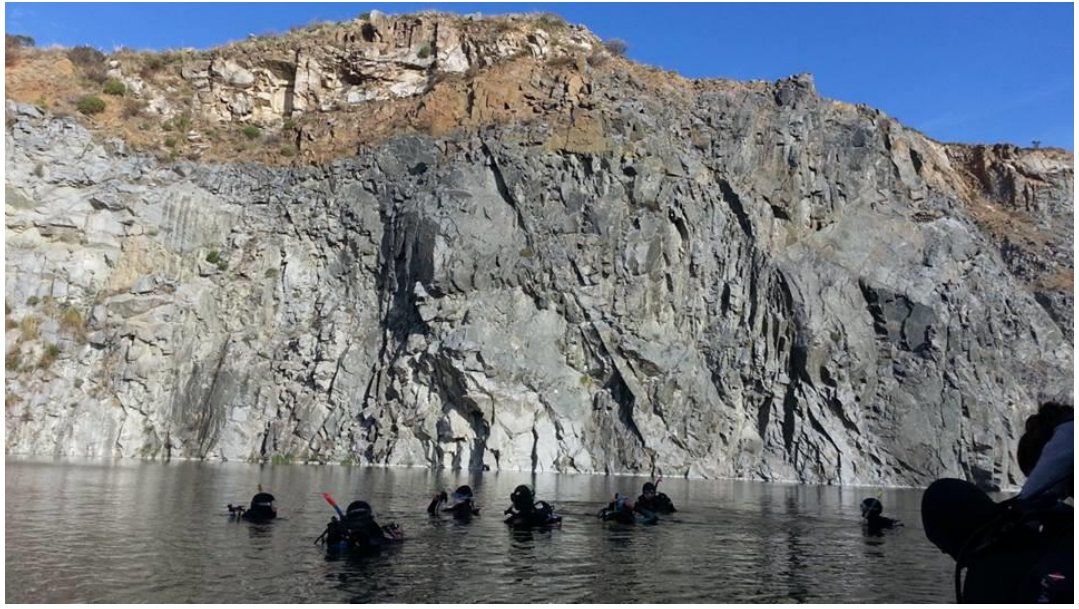

Imagen 8: Vista de unas cavas inundadas donde se practica buceo.

Fuente: https://es-la.facebook.com/Cantera-de-Buceo-LA-SIRENA-Tandil-568199420020628/ 

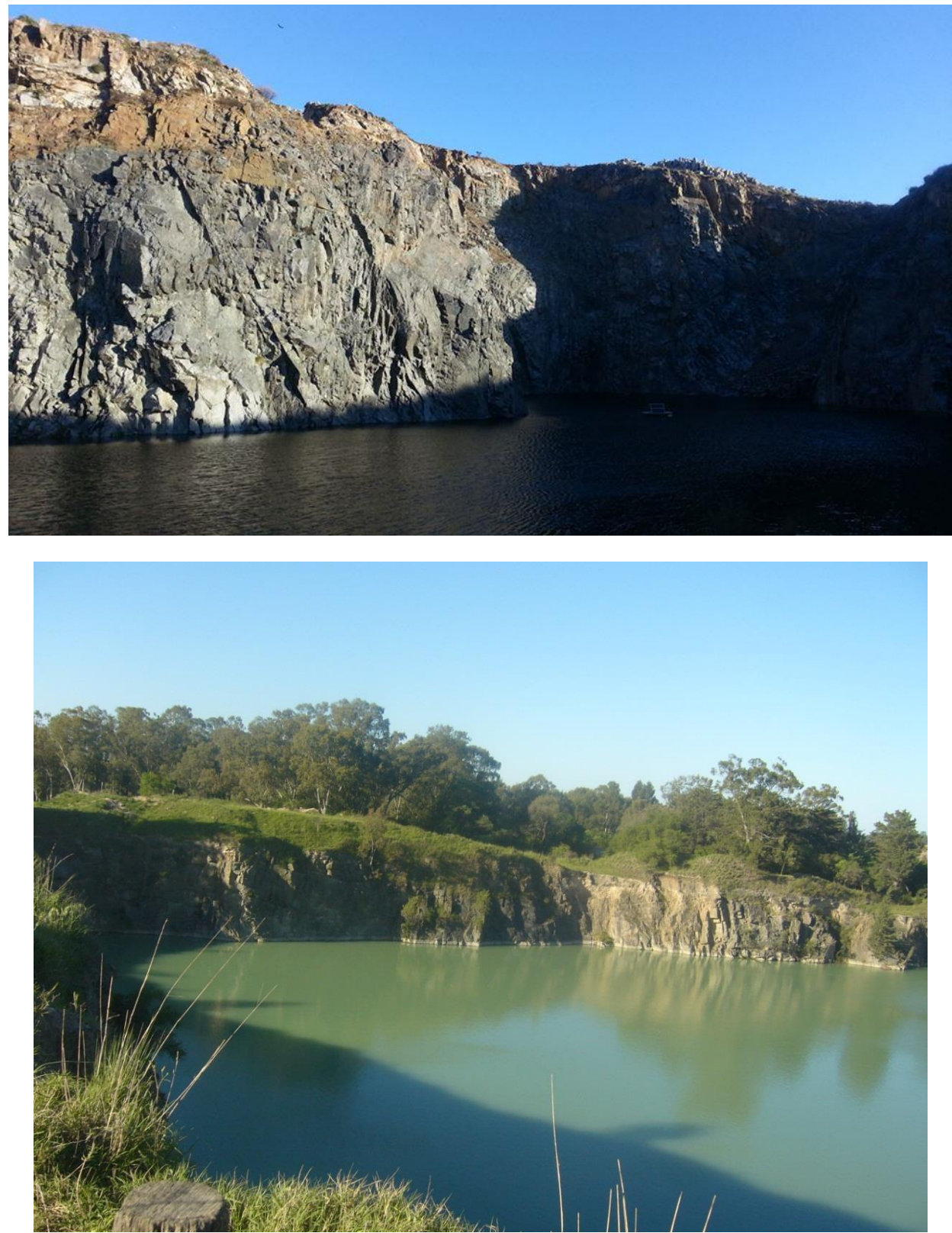

Imagen 9 y 10: Vista de otra cava inundada donde no hay actividad pero que podría utilizarse para el coasteering Fuente: Archivo personal. Año 2014 y 2016

El valor potencial del uso de estos espacios se acrecienta aún más si consideramos que Argentina en general no posee este tipo de costas, principalmente de fiordos. El litoral de la región pampeana es de llanura y el de la Patagonia es básicamente de acantilados, implicando situaciones extremadamente difíciles para desarrollar esta práctica de turismo aventura. Si existen algunas ofertas de coasteering en algunos de los lagos andinopatagónicos y en ríos de la misma región.

Por último, es importante considerar que en el destino se ha comenzado a capacitar en actividades de turismo aventura. En este sentido surgió una Diplomatura Universitaria en Turismo Alternativo con especialización en técnicas de escalada y rapel organizado por la Facultad de Ciencias Humanas de la UNICEN, donde se dicta la Licenciatura en turismo, junto a la Dirección de Turismo del Municipio de Tandil y el Instituto Mixto de Turismo organismo integrado por el sector público y privado, estos últimos interesados fuertemente en capacitar y mejor la calidad del servicio turístico. Es así que esta propuesta surge a partir de una demanda de múltiples actores vinculados a las actividades turístico-recreativas, buscando así aportar profesionalismo y mayor calidad e intentando unificar criterios respecto a las actividades orientadas a escalada y trekking que se realizan actualmente en el destino. Esto permite tener una base de conocimientos y de formación que podrían aprovecharse para acrecentar la demanda con estas nuevas modalidades. 


\section{REFLEXIONES FINALES}

A modo de reflexión final podemos decir que el destino turístico evaluado cuenta con condiciones físicas (geomorfología del sector serrano y áreas mineras con presencia de bloques de rocas de buen tamaño y cavas inundadas), de mercado (un porcentaje de los turistas que concurren al destino es para realizar distintos tipos de actividades de turismo aventura aunque no puede precisarse el número) y de recursos humanos (existe personal capacitado y se están generando ofertas educativas que permitan fortalecer la calidad de los servicios brindados) que podrían potencialmente utilizarse para el desarrollo de las modalidades de turismo aventura, denominadas bouldering y coasteering. Este desarrollo podría permitir generar un producto innovador orientado para un nicho de mercado creciente, ya que cada vez son más las personas que buscan practicar actividades que impliquen un esfuerzo físico importante (ejemplo de esto es el surgimiento del crossfit) pero con un componente lúdico y de aventura, con emociones fuertes.

Pero el desarrollo de estas actividades de turismo aventura no puede ser espontaneo y descontrolado, como ha ocurrido con muchas otras actividades, deben estar acompañadas de estudios específicos del medio natural, tanto para evitar impactos ambientales, incluso en áreas que ya fueron transformadas, como para reducir las posibilidades de accidentes, ya que lamentablemente la falta de planificación y organización ha generado situaciones graves que incluyeron víctimas fatales (por ejemplo accidentes en actividades de buceo y en recorridos con cuatriciclos).

En este sentido es necesario que el sector público (Dirección de Turismo e Instituto Mixto de Turismo) no sea solo promotor de las actividades, sino que asuma un rol de organizador a nivel territorial y de ente de control, fundamentalmente en las habilitaciones que se otorgan. Esto es fundamental si el destino pretende posicionarse como un centro de turismo aventura, aunque esta se superpone a otras prácticas como el turismo religioso, lo cual dificulta visualizar la imagen del destino. Esta variedad, si bien en ocasiones es positiva por atraer a distintos y muy diversos mercados, también puede ser dificultad para la gestión y planificación de las actividades.

Por otro lado, una de las ventajas del desarrollo de estas modalidades es que podrían revalorizarse espacios mineros que actualmente se encuentran abandonados y que se han convertido en áreas que generan graves riesgos desde el punto de vista de la seguridad para la población local principalmente e incluso en focos potenciales de contaminación a partir de la acumulación de residuos, lo cual es sumamente difícil de controlar. El bouldering y el coasteering podrían transformarlos en puntos de recepción de turistas otorgándoles un nuevo papel dentro del desarrollo territorial de la ciudad y del área circundante y si bien esto no implicaría que no se pueda acceder para realizar otras actividades, permitiría generar nuevas situaciones de control por parte del sector privado puesto que necesita mantener la belleza escénica y la calidad del paisaje para atraer a los turistas.

Finalmente se podría diversificar la oferta en general del destino, pero también de la oferta específica del turismo aventura posicionando a Tandil en la región e inclusive a nivel nacional a partir de un segmento que está creciendo, tal vez más lento que en los países desarrollados, pero que va en aumento de todas formas y requiere de nuevos espacios para su desarrollo.

\section{BIBLIOGRAFÍA}

Anthony R. (2011) An Investigation into Perceptions of Coasteering in the Pembrokeshire Coast National Park Amongst Participants in Other Forms of Recreation. Postgraduate Course in Sustainable Communities and Environments Dissertation. Sheffield Hallam University.

Araújo Vila, N., V. Paül Carril, J. Fraiz Brea (2011). El turismo activo o de aventura como componente destacado del turismo de naturaleza. Análisis de la oferta en Galicia. Gran Tour: Revista de Investigaciones Turísticas. Murcia. № 4 pp.8-31.

ATTA https://www.adventuretravel.biz/research/2016-adventure-tourism-development-index/ (Sitio revisado en junio de 2016)

ATTA: http://www.fairtrade.travel/uploads/files/ATTA_Adventure_Tourism_Market_Study_2013. (Sitio revisado en mayo de 2013)

Britto de Azevedo M., F y S. Alencar de Farias (2013) Riesgos ¿Qué riesgos? Estrategias de reducción de riesgos y participación emocional de consumidores de turismo aventura. En Estudios y Perspectivas en Turismo. Vol 22, 65-83.

Fanchini, M.; Violette, F. Impellizzeri, F. M.; Maffiuletti, N. (2013) Differences in Climbing-Specific Strength Between Boulder and Lead Rock Climbers. Journal of Strength \& Conditioning Research. Volume 27 Issue 2 - p 310-314 
Fernández Alvariño, C. (2010) Sociedad postmoderna y turismo. Nuevas tendencias de ocio (Postturismo). Ponencia del X Congreso de la Federación Española de Sociología. Pamplona. http://www.fesweb.org/congresos/X/index.php

Fernández, G., y A. Ramos (2015) Tandil ¿un destino de aventura? Un análisis de las características de la oferta Revista Realidad, Tendencias y Desafíos en Turismo. CONDET Año XV Volumen 13.

Hall, C.M. (1989) Special Interest Travel: A Prime Force in teh Expansion of Tourism? In R. Welch. (ed). 81-89. Geography in Action. Dunedin: University of Otago.

Silva, F. y M. C. T. Almeida (2011) Caracterização e potencialidades do coasteering em Portugal. Journal of Tourism and Sustainability. Volume I - Nr. 2 - Pages: 27-35

South West Watersports (2004). Te ofcial guide to water-sports. Coasteering Exeter: Marine Southwest Taskforce. (URL: http:// www.sw-tersports.com, consultado a 2011-03-02).

Sung, H.H. (1996) Definition of Adventure Travel: Conceptual Framework for Empirical Application. Unpublished Master's Thesis, Purdue University, West Lafayette, IN.

Tyler-Walters, H., (2005) Assessment of the Potential Impacts of Coasteering on Rocky Intertidal Habitats in Wales. Report to Cyngor Cefn Gwlad Cymru / Countryside Council for Wales from the Marine Life Information Network (MarLIN). Marine Biological Association of the UK, Plymouth.

White, D J; Olsen, P. (2010) A Time Motion Analysis of Bouldering Style Competitive Rock Climbing. Journal of Strength \& Conditioning Research. Volume 24 - Issue 5 - pp 1356-1360. 